\title{
(6) OPEN ACCESS \\ A pilot survey of junior doctors' attitudes and awareness around medication review: time to change our educational approach?
}

\author{
Barry Jubraj, ${ }_{1}^{1,2}$ Vanessa Marvin, ${ }^{1,2}$ Alan J Poots, ${ }^{2}$ Shreena Patel, ${ }^{3}$ Iñaki Bovill, ${ }^{4}$ \\ Nina Barnett, ${ }^{5}$ Laurel Issen, ${ }^{2}$ Derek Bell ${ }^{2}$
}

\begin{abstract}
- Additional material is published online only. To view please visit the journal online (http://dx.doi.org/10.1136/ ejhpharm-2015-000664)

'Pharmacy Department, Chelsea and Westminster Hospital NHS Foundation Trust, London, UK

${ }^{2}$ National Institute of Health Research (NIHR) Collaboration for Leadership in Applied Health Research and Care Northwest London (CLAHRC $\mathrm{NWL}$ ), London, UK ${ }^{3}$ Department of Pharmacy, King's College London, London, UK

${ }^{4}$ Department of Medicine for the Elderly, Chelsea and Westminster Hospital NHS Foundation Trust, London, UK ${ }^{5}$ Pharmacy Department, London North West Healthcare NHS Trust (Northwick Park Hospital), London, UK
\end{abstract}

\section{Correspondence to} Dr Alan J Poots, National Institute of Health Research (NIHR) Collaboration for Leadership in Applied Health Research and Care Northwest London (CLAHRC NWL), London SW10 9NH, UK a.poots@imperial.ac.uk

Received 12 February 2015 Revised 8 April 2015 Accepted 30 April 2015 Published Online First 3 June 2015

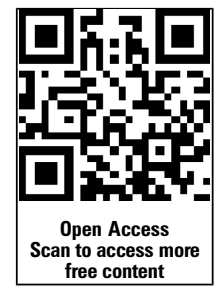

CrossMark

To cite: Jubraj $B$, Marvin $\mathrm{V}$, Poots AJ, et al. Eur J Hosp Pharm 2015;22:243-248.

\section{ABSTRACT}

Objectives Our aim was to explore junior doctors' attitudes and awareness around concepts related to medication review, in order to find ways to change the culture for reviewing, altering and stopping inappropriate or unnecessary medicines. Having already demonstrated the value of team working with senior doctors and pharmacists and the use of a medication review tool, we are now looking to engage first year clinicians and undergraduates in the process.

Method An online survey about medication review was distributed among all 42 foundation year one (FY1) doctors at the Chelsea and Westminster Hospital NHS Foundation Trust in November 2014. Descriptive statistics were used for analysis.

Results Twenty doctors completed the survey (48\%). Of those, 17 believed that it was the pharmacist's duty to review medicines; and 15 of 20 stated the general practitioner (GP). Sixteen of 20 stated that they would consult a senior doctor first before stopping medication. Eighteen of 20 considered the GP and consultant to be responsible for alterations, rather than themselves. Sixteen of 20 respondents were not aware of the availability of a medication review tool. Seventeen of 20 felt that more support from senior staff would help them become involved with medication review.

Conclusions Junior doctors report feeling uncomfortable altering mediations without consulting a senior first. They appear to be building confidence with prescribing in their first year but not about the medication review process or questioning the drugs already prescribed. Consideration should be given to what we have termed a 'bottom-up' educational approach to provide early experience of and change the culture around medication review, to include the education of undergraduate and foundation doctors and pharmacists.

\section{INTRODUCTION}

The UK Department of Health quotes a definition of medication review as 'A structured, critical examination of a patient's medicines with the objective of reaching an agreement with the patient about treatment, optimising the impact of medicines, minimising the number of medication-related problems and reducing waste'. ${ }^{1}$ Medication review, including 'deprescribing' inappropriate or unnecessary medicines should be part of routine prescribing practice in all healthcare settings; particularly in older populations given that problematic prescribing is estimated to occur in more than one-third of over 70 year olds. ${ }^{2} 3$ The setting for medication review includes secondary care (hospitals), where doctors in their first year of practice (known in the UK as 'foundation year one' or 'FY1') are involved in prescribing and dosing adjustments particularly for acutely unwell patients. These patients may have come into the hospital without their regular medicines or an up-to-date list, leading to delays or unsafe treatment. Unreconciled medicines compromise optimum review of medicines in the acute setting (V Marvin et al, unpublished data, April 2015). In addition, medication-related problems may have contributed to the presenting complaint, or an adverse drug reaction could be the cause of hospitalisation. ${ }^{4}$

Urgency and complexity of overall care make identifying potentially inappropriate medications as part of a medication review a significant challenge in the acute setting. Locally, the medicines policy for our hospital contains standards for good medicines management including prescribing. Many British hospitals follow the Royal Pharmaceutical Society standards for hospital services where it is stated that on admission, or at first contact with the patient, medicines should also be reviewed for clinical appropriateness and to identify patients in need of further pharmacy support. Moreover, the pharmacy team should provide the leadership, systems support and expertise that enable a multiprofessional team to do this as part of the admission process. $^{5}$

However, reviewing and stopping medicines do not appear to be part of the culture of routine practice and generally not part of the admitting (junior) doctor's process. There is evidence that at the early stages of a medical career, doctors may not possess sufficient competence and confidence to perform a medication review, ${ }^{6}$ and may feel uncomfortable stopping medicines that more senior doctors have initiated; indicative perhaps of hierarchical dominance. Research is needed to explore the ability and attitudes of junior doctors in contributing to medication review, as well as their awareness of medication review tools that have been developed to aid them in this process. This will allow us to identify barriers to the contribution of FY1 doctors to managing medicines in the acute setting, where they are regular prescribers if not 'deprescribers'.

The National Institute of Health Research (NIHR) Collaboration for Leadership in Applied Health Research and Care Northwest London (CLAHRC NWL) is a programme hosted by the 
Chelsea \& Westminster Hospital NHS Foundation Trust (CWFT). Their remit is to translate research into practice, and find how to best embed the changes made. Early CLAHRC NWL improvement projects led to interventions involving pharmacists working closely with junior doctors to facilitate accurate, reliable medicines reconciliation at admission to hospital and through to discharge. Further work included adapting the original Screening Tool of Older Persons potentially inappropriate Prescriptions (STOPP) medication review tool, ${ }^{7}$ through which it was found that $1331 / 1368$ older patients (97\%) were prescribed at least one medicine indicated as a potential problem. ${ }^{8}$ This tool was developed into the 'STOPIT' tool used at CWFT, to provide structure and facilitate the medication review process for all clinicians with particular effectiveness demonstrated in the elderly rehabilitation setting. ${ }^{9}$

Using the action-effect method (a quality improvement method) ${ }^{10}$ (see online supplementary appendix), a need for research into the role of junior doctors in medication review was identified as part of CLAHRC NWL's overall medicines optimisation themes. NIHR CLAHRC NWL brought together stakeholders interested in medication review in the elderly acute care setting and facilitated a session to develop a shared aim for collaboration, the achievement of which would be contingent on finding an effective, reliable and embedded process for reviewing and optimising medicines within all settings. The project team used the action-effect method to develop causeeffect chains to influence these factors, highlighting a need to develop and embed a culture where optimising medicines for an individual patient is the norm. We postulated that junior doctors require the development of skills, confidence and awareness to contribute to the medication review process. This article discusses the further analysis of the survey results, some of which were presented at a recent conference. ${ }^{11}$

\section{Objectives}

1. To elicit junior doctors' attitudes to and awareness of the need to review medicines in elderly patients.

2. To explore factors that may hinder medication review and deprescribing.

3. To use this information to formulate a 'bottom-up' approach to educate foundation and undergraduate doctors and pharmacists in preparation for their roles in medication review.

\section{METHOD}

Ethics approval was sought from CWFT Research and Development and National Research Ethics Service (NRES) but was deemed not required as this was a service evaluation.

Our study comprised an online questionnaire survey that investigated the attitudes of all CWFT FY1 doctors to concepts around medication review and their familiarity with medication review tools. The survey was phrased to avoid confusion between 'medication review' and 'medicines reconciliation'. A focus group of two senior CWFT/CLAHRC pharmacists, a consultant geriatrician, two FY1 doctors and an undergraduate pharmacy student used the action-effect diagram to identify survey themes.

Thirteen questions were derived from the focus group themes, and circulated among the group for further comments before piloting in October 2014. The survey was designed in the online survey tool SurveyMonkey; where a question offered an 'other' response to tick, with a free text box provided to allow for any additional comments.

The final postpilot survey comprised 16 questions and was distributed to all 42 FY1 doctors within CWFT between 27
Table 1 The frequency of medication review undertaken by foundation year one (FY1) doctors on their elderly patients

\begin{tabular}{llllll}
\hline Frequency & Daily & Every ward round & Weekly & Never & Other \\
\hline Responses & 6 & 5 & 3 & 1 & 5 \\
\hline
\end{tabular}

October 2014 and 23 November 2014. An incentive was offered in the form of a voucher drawn at random from participating doctors. Strategies for improving survey response rate were developed iteratively using plan-do-study-act (PDSA) cycles. This is an improvement science methodology facilitated by CLAHRC NWL to plan, predict, record and adapt our methodology through a series of methodical pilot studies. ${ }^{12}$

Descriptive statistics were used for frequency of responses and where appropriate cross-tabulations were applied. Given the sample size, no inferential statistics were applied, with a focus given to qualitative aspects to highlight areas in which training interventions can be made. The results for this are broken down by theme: specialty of the FY1, medication review, medication review tools, feelings around prescribing and stopping medication and what would help.

\section{RESULTS}

Between 28 October 2014 and 21 November 2014, 12 online responses were received. Using PDSA methodology, the team gathered at a CWFT weekly junior doctor training session on 21 November 2014 and distributed paper copies of the survey which yielded a further eight responses. The survey was closed on 23 November 2014, with a response rate of 20/42 FY1 doctors (48\%). Eleven of $20(55 \%)$ respondents were working in medicine, six in surgery $(30 \%)$ and three $(15 \%)$ in acute medicine.

\section{Medication review}

Table 1 states the self-reported frequency of medication review undertaken by FY1 doctors on their elderly patients. 'Other' (free text) responses included (frequency in brackets): once per admission (2), when the patient has a prolonged stay (1), if asked by a consultant/pharmacy/need something new (1) and every 2-3 days (1).

The opinions of FY1 doctors on who reviews and stops medication are shown in tables 2 and 3, respectively. Reviewing elderly patient's medication is mostly commonly thought to be carried out by the pharmacist, followed by general practitioner (GP), specialist registrar (SpR) and consultant. All three 'other' responses in table 2 stated the FY1 doctor.

The majority of participants chose GP and consultant for being the professionals they believe are responsible for stopping elderly patient's medication. The $\mathrm{SpR}$ was the next most popular option, followed by senior house officer (SHO), FY1 and pharmacist.

Table 2 Responses to 'who reviews elderly patient's medication?' (multiple options permitted)

\begin{tabular}{llllll}
\hline Professional & Pharmacist & GP & SpR & Consultant & Other* \\
\hline Responses & 17 & 15 & 11 & 10 & 3 \\
\hline GP, general practitioner; SpR, specialist registrar.
\end{tabular}


Table 3 Responses to 'who stops elderly patient's medication?' (multiple options permitted)

\begin{tabular}{lllllll}
\hline Professional & Consultant & GP & SpR & SHO & FY1 & Pharmacist \\
\hline $\begin{array}{l}\text { Responses } \\
18\end{array}$ & 18 & 15 & 14 & 9 & 8 \\
\hline $\begin{array}{l}\text { FY1, foundation year one; GP, general practitioner; SHO, senior house officer; SpR, } \\
\text { specialist registrar. }\end{array}$
\end{tabular}

Within their current specialty, most FY1 doctors reported stopping medications weekly (11), with no respondents reporting 'never'. Outside their specialty; however, FY1s reported either never stopping medicines (7) or only do so monthly (6). No FY1s reported stopping medications daily (figure 1).

\section{Medication review tools}

Sixteen out of 20 FY1 doctors reported having never heard of a medication review tool. Three of the remaining four doctors stated having heard of the STOPIT tool (see the Introduction) and one had heard of STOPP/START. ${ }^{13}$ When questioned if the FY1s had ever used the selected tool in clinical practice, only one respondent stated yes.

\section{Feelings around prescribing and stopping medication}

Sixteen out of 20 FY1 doctors reported feeling comfortable prescribing within their current specialty. The same number of doctors also stated feeling uncomfortable stopping or starting a medication without speaking to a senior first.

No respondent reported feeling 'very uncomfortable' prescribing within their specialty; however, 10 FY1 doctors reported feeling uncomfortable with prescribing medication outside of their current specialty.

Eighteen out of $20 \mathrm{FY} 1 \mathrm{~s}$ stated having questioned the suitability of a medicine for an elderly patient; the others reporting that they had not done this. After questioning the medication, the FY1s working in medicine (following analysis by speciality) were more likely to speak to their $\mathrm{SHO} / \mathrm{SpR} /$ consultant (figure 2).

Seventeen out of 20 respondents would approach a pharmacist if they did not recognise a medication prescribed for their elderly patient. This was followed by registrars (12 responses). 'Other' responses included (frequency in brackets): British
National Formulary (4) and Foundation Year Two doctors (2) (figure 3).

\section{What would help with confidence in medication review?}

When asked what would help FY1 doctors become more confident in making suggestions to review/stop medicines, 17/20 participants chose 'support from senior doctors', followed by 10/20 selecting 'my team being more open to medication review'. Nine out of 20 equally stated that support from pharmacists and a medication review tool would be of help. Finally, 7/20 reported that more teaching on the subject in the medical school syllabus would help improve their confidence in stopping and reviewing medicines.

\section{DISCUSSION}

Our FY1 survey is an early contribution to the investigation of FY1 doctors' views about medication review and awareness of medication review tools. Although the sample size was small, with a possibility of responder-selection bias given the $48 \%$ response rate, the results were as the project team expected, suggesting that if FY1s do undertake medication review, it is likely to be speciality specific and any further review is likely to require support from seniors. If FY1s have questions about whether to review or stop medicines, their seniors would be consulted, rather than their pharmacists, to whom they would address specific questions about unfamiliar medicines. Confidence and competence of FY1s in undertaking medication review may be a reason for not undertaking full reviews, and the majority of FY1s were not aware of medication review tools 'STOPIT' and 'STOPP'. 713

There may be a number of reasons why FY1 doctors may not perform routine, complete medication reviews. It could be that they lack familiarity with the concept or definition of medication review, ${ }^{1}$ or confidence in using medication review tools. We have also discussed locally the expectations around whether a medication review is required. For example, local focus groups suggested that indications for medicines are frequently not known to the admitting team, medication lists may not be up to date, and surgical teams may be reluctant to change medications. ${ }^{9}$ These factors may give the message to junior doctors that a medication review is not needed or is impractical. Moreover, junior doctors may lack the required competence in
Figure 1 Responses to 'with what frequency do you stop medicines?' within and outside the specialty to which currently attached.

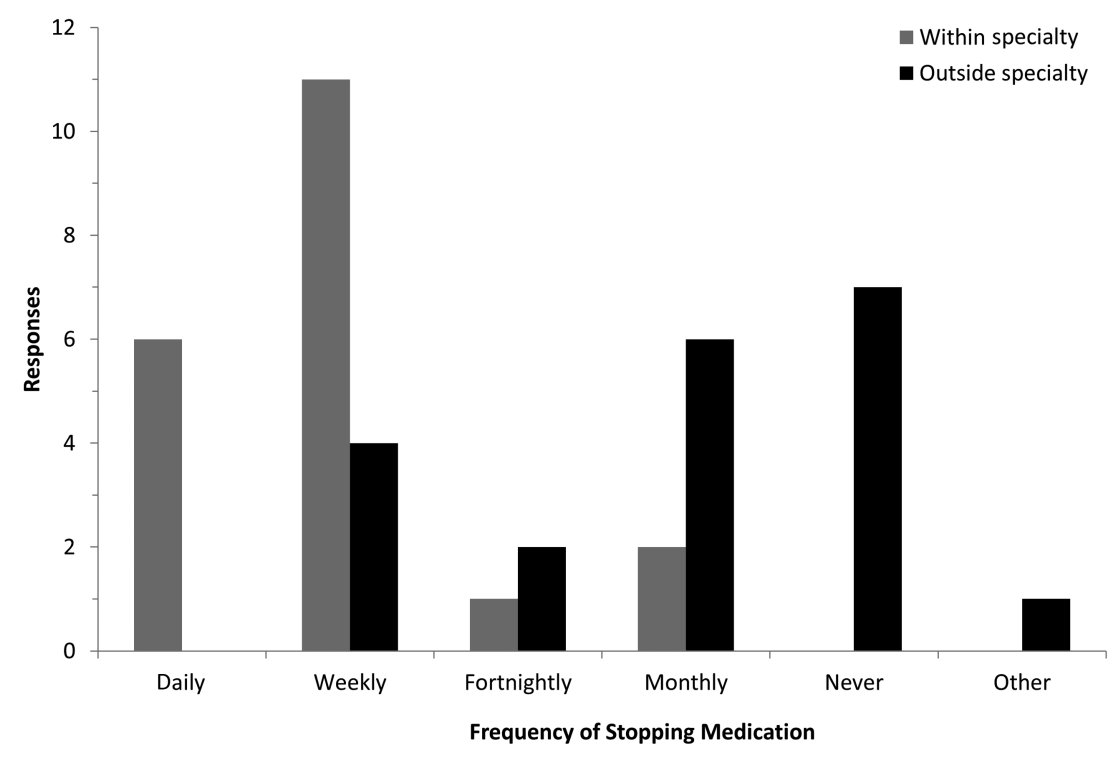


Figure 2 For those answering 'yes' $(18 / 20)$ to 'have you questioned in your mind whether a medicine for one of your elderly patients is necessary?' This chart shows the responses to 'what did you do about it?' SHO, senior house officer; SpR, specialist registrar.

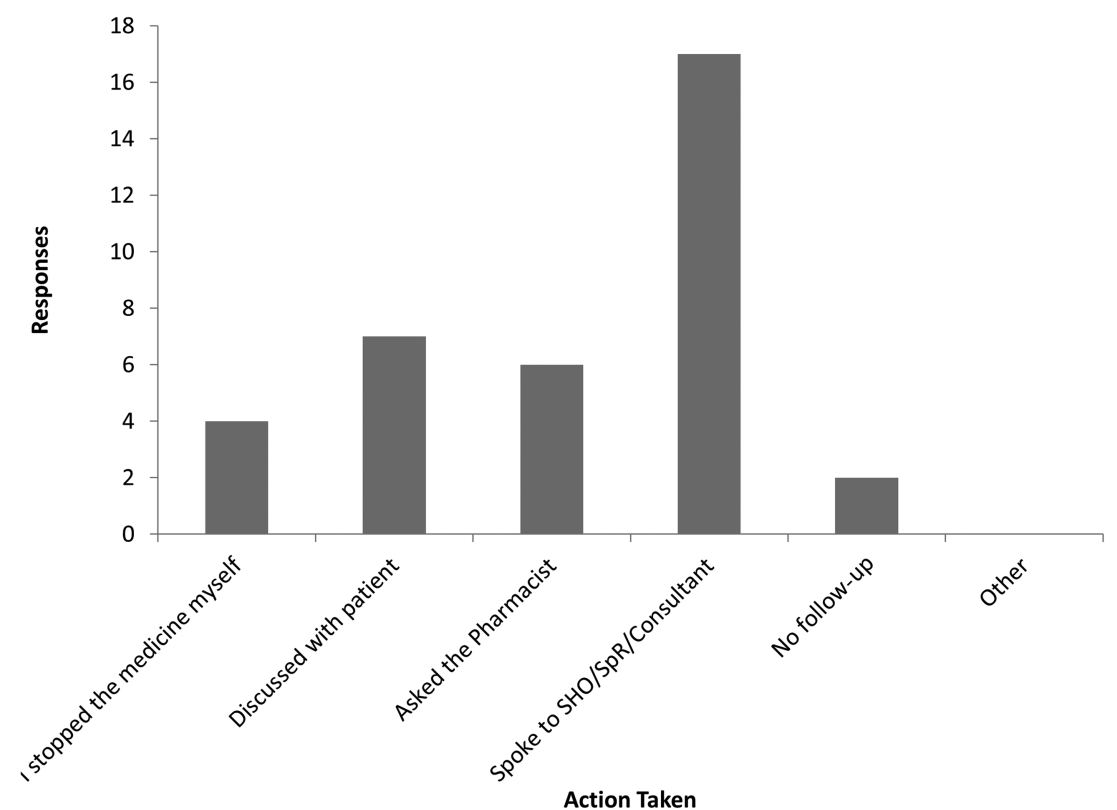

evaluating medication across a range of multimorbidities in older people. It may also be that senior staff, when undertaking medication review, do not do it in the presence of the junior doctors and/or do not share their methodology or rationale, making the process of structured medication review invisible to junior doctors and preventing learning. These factors could conspire so as to reduce opportunities to gain experience, and with past events guiding future behaviours. ${ }^{14}$ This may impact the capability to perform such review tasks. In addition, FY1s may believe that medication review is the responsibility of more senior staff and thus they do not need to be involved, or have a reluctance to review and optimise (including appropriate deprescribing) what senior doctors, including GPs, have previously prescribed. In this respect, we consider that hierarchical dominance is a theme worthy of further exploration. The aviation industry suffered a number of catastrophic events that led to changes whereby hierarchy is maintained; but a less authoritarian stance is sought to make optimum use of all available resources, including people, to promote safety and efficiency. ${ }^{15}$ This provides a supportive learning opportunity within a multidisciplinary team to gain experience. This approach, through a number of iterations, has been used within the healthcare settings. ${ }^{16-18}$ As we apply these principles, we propose that a 'bottom-up' approach to medication review may begin the change of culture and acquisition of experience that engages doctors and pharmacists at the undergraduate and foundation levels in the medication review process. Seven out of 20 of our FY1 respondents supported the introduction of relevant undergraduate education.

\section{Study limitations and future work}

Although our survey is not validated and this study is exploratory in nature, the purpose was to begin to elucidate our FY1 doctors' attitudes to and awareness around specific questions relating to the concept of medication review. For example, one question was 'Have you questioned in your mind whether a
Figure 3 Responses to 'who would you ask if you see a medication prescribed for your elderly patient that you don't recognise?' (multiple options permitted).

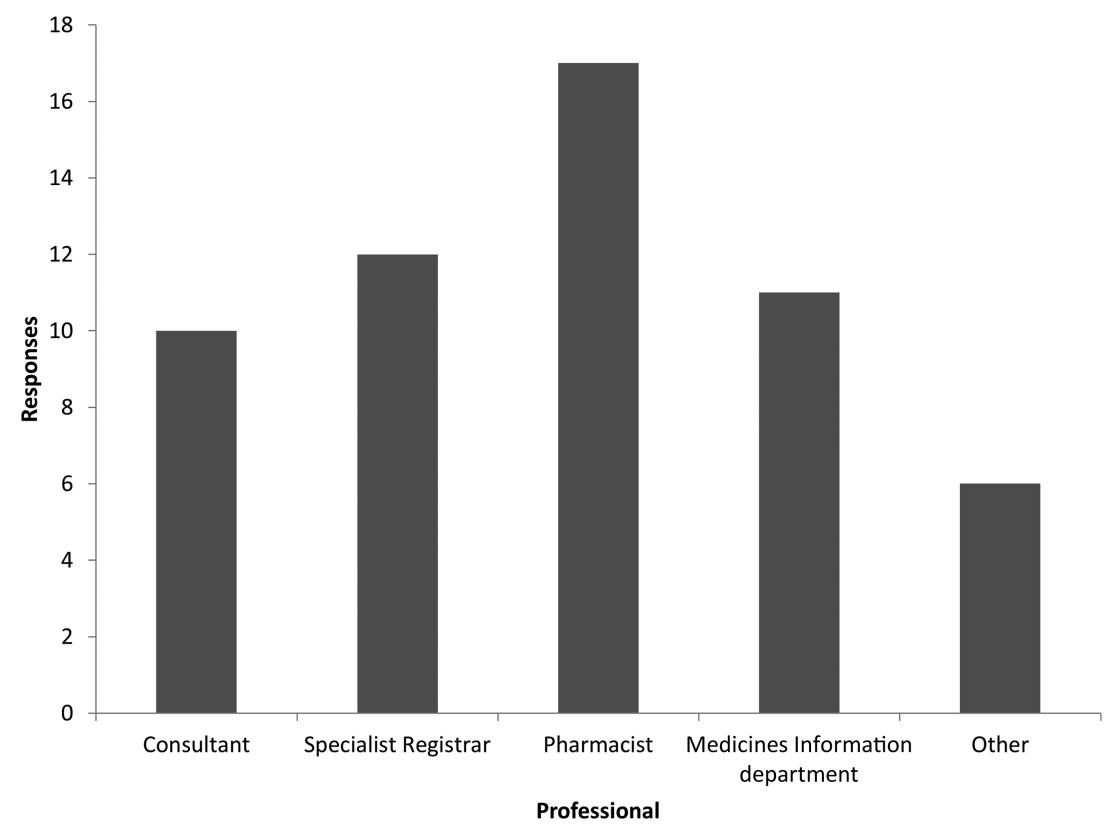

Jubraj B, et al. Eur J Hosp Pharm 2015;22:243-248. doi:10.1136/ejhpharm-2015-000664 
medicine for one of your elderly patients is necessary?' Little literature, if any, elucidates the views of junior doctors around concepts related to medication review. This FY1 survey begins to explore this and we plan to repeat the medication review survey for all FY1 doctors in London, given the generalisability of our work and the need to ensure that non-responder and social desirability bias are properly evaluated.

The survey results are useful as we consider the education of pharmacists and doctors at CWFT. Moreover, through discussion of the findings of our projects within NIHR CLAHRC NWL and with our junior doctors, we are in 2015 seeking to launch a 'bottom-up' approach to engaging doctors and pharmacists in the medication review process in all settings, including acute care. We hope to influence undergraduate and foundation education for doctors and pharmacists in London and possibly beyond, a concept that has recently been supported outside Europe. ${ }^{19}$ Our approach is firmly based on the principle that appropriate medication review must be based on adequate medicines reconciliation (V Marvin et al, unpublished data, April 2015), and our local work supports the notion that interdisciplinary medication review is an ideal way to review medication. ${ }^{9}$ We also believe that the use of medication review tools can equip junior doctors and pharmacists to participate in the review process by prompting senior clinicians in the acute setting and after discharge from hospital. This may support GPs in managing their primary care patients with clear, concise and complete information about medication review undertaken during a hospital admission. We are acutely aware of the need to ensure and teach novice practitioners not to deprescribe indiscriminately, but prompt their seniors for support, having identified possible medicines to review. Educating undergraduate and foundation doctors and pharmacists will also prepare them for their responsibilities as seniors around appropriate prescribing and deprescribing, not least through gaining experience. To this end, we have begun to influence the undergraduate curriculum in one pharmacy school by running final year medication review case studies, and are suggesting medication

\section{What this paper adds}

What is already known on this subject

- Much research and opinion-based literature around appropriate prescribing, 'deprescribing' and medication review is being published; but most is aimed at senior doctors and pharmacists.

- Little has been published about the views and awareness of medication review among junior doctors, pharmacists and students.

\section{What this study adds}

- Our motivation for undertaking this pilot study was based on work in Northwest London (NWL) to embed a culture of medication review and concomitantly identify the need to influence undergraduate and newly qualified doctors and pharmacists.

- We present here a pilot study of junior doctors' views on and awareness of concepts around medication review in one UK hospital.

- Our survey has led to a suggested 'bottom-up' approach to education about medication review, which we believe to be the first of its kind and is being developed currently in London. review-related learning outcomes for the London-wide and national foundation curricula for pharmacists. Our next steps are to emulate this activity for medical schools and foundation doctor training.

\section{CONCLUSION}

We encourage our European colleagues to explore the ability of and any barriers to junior doctors and pharmacists to contribute to the medication review process in the acute setting, and to ensure that they understand the imperative of adequate medicines reconciliation, where appropriate for their health system, before undertaking a medication review.

Acknowledgements The authors would like to thank Dr Muhammad Umer and Dr Sarah-Jane Trehane (FY1 doctors) for helping prepare and distribute the survey at CWFT. We are also grateful to the FY1 doctors at CWFT who completed the survey for us. Publishing costs are supported by Imperial College London Central Library.

Disclaimer This article presents independent research commissioned by the National Institute for Health Research (NIHR) under the Collaborations for Leadership in Applied Health Research and Care (CLAHRC) programme for Northwest London (NWL). The views expressed in this publication are those of the author(s) and not necessarily those of the NHS, the NIHR or the Department of Health.

Competing interests None declared.

Ethics approval Chelsea and Westminster Hospital Research and Development.

Provenance and peer review Commissioned; externally peer reviewed.

Data sharing statement All the data collected via SurveyMonkey are available to the coauthors via a login that is held by NIHR CLAHRC NWL.

Open Access This is an Open Access article distributed in accordance with the Creative Commons Attribution Non Commercial (CC BY-NC 4.0) license, which permits others to distribute, remix, adapt, build upon this work non-commercially, and license their derivative works on different terms, provided the original work is properly cited and the use is non-commercial. See: http://creativecommons.org/ licenses/by-nc/4.0/

\section{REFERENCES}

1 Department of Health Publications, Policy and Guidance. http://webarchive. nationalarchives.gov.uk/+/www.dh.gov.uk/en/publicationsandstatistics/publications/ publicationspolicyandguidance/browsable/dh_5354376 (accessed 7 Apr 15).

2 Cahir C, Fahey T, Teeling M, et al. Potentially inappropriate prescriptions and cost outcomes for older people. Br J Clin Pharmacol 2010;69:543-52.

3 Hamilton H, Gallagher P, Ryan C, et al. Potentially inappropriate medications defined by STOPP criteria and the risk of adverse drug events in older hospitalized patients. Arch Intern Med 2011;171:1013-19.

4 Pirmohamed $M$, James $S$, Meakin $S$, et al. ADRs as a cause of admission to hospital: prospective analysis of 18,820 patients. BMJ 2004;329:15-19.

5 Royal Pharmaceutical Society: Professional Standards for Hospital pharmacy Services: Optimising Patient outcomes from Medicines v 2 July 2014. http://www. rpharms.com/unsecure-support-resources/ professional-standards-for-hospital-pharmacy.asp? (accessed 7 Apr 15)

6 Keijsers C, van Doorn A, van Kalles A, et al. Structured pharmaceutical analysis of the systematic tool to reduce inappropriate prescribing is an effective method for final-year medical students to improve polypharmacy skills: a randomized controlled trial. J Am Geriatr Soc 2014;62:1353-9.

7 Gallagher P, O'Mahony D. STOPP (Screening Tool of Older Persons potentially inappropriate Prescriptions) application to acutely ill elderly patients and comparison with Beers criteria. Age Ageing 2008;37:673-9.

8 Thakkar K, Jacklin A, Patel N, et al. The improving prescribing for the elderly project (ImPE, NIHR CLAHRC NWL). The Health Foundation, 2013.

9 Abdul-Saheb M, Jubraj B, Bovill I, et al. Intermediate care: an optimal setting for review of inappropriate medication in elderly patients? Geriatr Med (http://www. gmjournal.co.uk) 2014;44:13-17.

10 Reed J, McNicholas C, Woodcock T, et al. Designing quality improvement initiatives: the action effect method, a structured approach to identifying and articulating programme theory. BMJ Qual Saf 2014;23:1040-8.

11 Patel $S$, Jubraj $B$, Marvin $V$, et al. A survey exploring junior doctors' attitudes to medication review [abstract in press]. Poster presentation at Prescribing and Research in Medicines Management (UK \& Ireland) (PRIMM) Conference; London, 2015.

12 Taylor M, McNicholas C, Nicolay C, et al. Systematic review of the application of plan-do-study-act method to improve quality in healthcare. BMJ Qual Saf 2014;23:290-8 


\section{Original article}

13 O'Mahoney D, O'Sullivan D, Byrne S, et al. STOPP/START criteria for potentially inappropriate prescribing in older people: version 2. Age Ageing 2015;44:213-18.

14 Ouellette JA, Wood W. Habit and intention in everyday life: the multiple processes by which past behaviour predicts future behaviour. Psychol Bull 1998;124:57-74

15 Helmreich RL, Merritt AC, Wilhelm JA. The evolution of Crew Resource Management training in commercial aviation. Int J Aviat Psychol 1999;9: $19-32$.
16 McConaughey E. Crew resource management in healthcare: the evolution of teamwork training and Med Teams. J Perinat Neonat Nurs 2008;22:96-104.

17 Sundar E, Sundar S, Pawlowski J, et al. Crew resource management and team training. Anesthesiol Clin 2007:25:283-300.

18 Vyt A. Interprofessional and transdisciplinary teamwork in health care. Diabetes Metab Res Rev 2008;24(Suppl 1):S106-9.

19 Scott IA, Hilmer SN, Reeve E, et al. Reducing inappropriate polypharmacy the process of deprescribing. JAMA Intern Med 2015;175:827-34. http://archinte. jamanetwork.com/article.aspx?articleid=2204035 (accessed 7 Apr 15). 